\title{
HACs with non-repetitive centromeres
}

Engineering human centromeres will allow versatile applications.

$\mathrm{T}$

ranslating success in yeast to success in humans is not trivial. The Synthetic Yeast 2.0 project successfully wrote the chromosomes of budding yeast, but replicating this feat and creating artificial human chromosomes (HACs) - the goal of the Human Genome Project-Write ran into challenges at the centromere. This chromosomal region that specifies attachment of the mitotic spindle and ensures proper segregation during cell division is short and manageable in yeast, but humans require much larger repeat regions that need CENP-A, a histone $\mathrm{H} 3$ variant, for the induction and maintenance of chromatin features that specify the centromeres. Relying on repetitive DNA makes HAC challenging to synthesize, assemble and maintain, since recombination at the repeats can lead to loss of essential sequences.

Ben Black, from the University of Pennsylvania, has made understanding centromere biology the focus of his research for more than a decade; his group has studied the biochemistry and structure of centromeres, as well as the epigenomic process needed for their establishment and maintenance. He came to the realization that "we won't really understand how they work until we can build them from scratch." He combined his lab's expertise with contributions from international collaborators to create HACs that do not need repetitive centromeric DNA.

Instead of replicating a canonical centromere in which CENP-A is recruited to the $\alpha$-satellite repeat sequences in the centromere via the repeat-binding CENP-B protein, the researchers directly recruited CENP-A nucleosomes to a repeat region on the HAC without the need for CENP-B. Then they went a step further and showed that the $\alpha$-satellite repeats were not required either and that HACs could be formed with non-repetitive DNA.

A more robust protocol to establish and maintain HACs, without the highly repetitive DNA that Black describes as a "nightmare to clone and handle at every step", will do more than allow the writing of human chromosomes. Black also foresees that the approach could be extended to other eukaryotes, such as plants and fungi different from budding yeast, as the pathway the researchers harnessed is conserved in many eukaryotes.

Nicole Rusk

Published online: 27 September 2019

https://doi.org/10.1038/s41592-019-0600-y

Research paper

Logsdon, G. A. et al. Human artificial chromosomes that bypass centromeric DNA. Cell 178, 624-639 (2019).

\section{CALL FOR PAPERS}

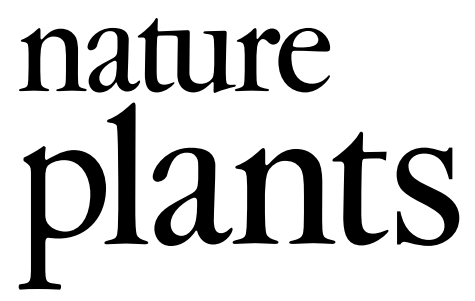

\section{From Bench to Biosphere}

Covers all aspects of plant science including evolution, genetics, development, interactions with the environment, and societal significance.

\section{Submit your research today www.nature.com/natureplants}
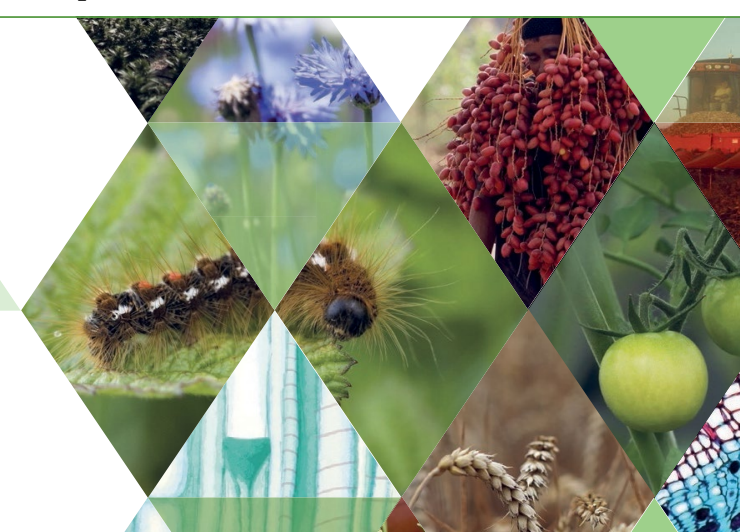